\title{
Intra-Pixel Gain Variations and High-Precision Photometry with the Infrared Array Camera (IRAC)
}

\author{
James G. Ingalls, Jessica E. Krick, Sean J. Carey, Seppo Laine, Jason A. Surace, William J. \\ Glaccum, Carl C. Grillmair, and Patrick J. Lowrance
}

\begin{abstract}
The Infrared Array Camera (IRAC) on the Spitzer Space Telescope has been used to measure $<10^{-4}$ temporal variations in point sources (such as transiting extrasolar planets) at 3.6 and $4.5 \mu \mathrm{m}$. Due to the under-sampled nature of the PSF, the warm IRAC arrays show variations of as much as $8 \%$ in sensitivity as the center of the PSF moves across a pixel due to normal spacecraft pointing wobble and drift. These intra-pixel gain variations are the largest source of correlated noise in IRAC photometry. Usually this effect is removed by fitting a model to the science data themselves (self-calibration), which could result in the removal of astrophysically interesting signals. We describe a new technique for significantly reducing the gain variations and improving photometric precision in a given observation, without using the data to be corrected. This comprises: (1) an adaptive centroiding and repositioning method ("Peak-Up") that uses the Spitzer Pointing Control Reference Sensor (PCRS) to repeatedly position a target to within 0.1 IRAC pixels of an area of minimal gain variation; and (2) the high-precision, high-resolution measurement of the pixel gain structure using non-variable stars. We show that the technique currently allows the reduction of correlated noise by almost an order of magnitude over raw data, which is comparable to the improvement due to self-calibration. We discuss other possible sources of correlated noise, and proposals for reducing their impact on photometric precision.
\end{abstract}

Keywords: methods: data analysis, space vehicles: instruments, techniques: photometric, planetary systems, observatories: Spitzer

\section{INTRODUCTION}

The Infrared Array Camera ${ }^{1}$ (IRAC) on board the Spitzer Space Telescope ${ }^{2}$ has been used to measure $<10^{-4}$ temporal variations in the fluxes of point sources at 3.6 and $4.5 \mu \mathrm{m} \cdot{ }^{3,4}$ Due to the undersampled nature of the PSF, the post-cryogenic ("warm") IRAC arrays show variations in measured photometry of as much as $8 \%$ (peak-to-peak) as the center of a source moves across a pixel with normal spacecraft pointing wobble and drift. ${ }^{5}$ Heater cycling in the spacecraft causes the relative angle between the Spitzer star tracker and the optical axes to vary cyclically with a period of $\sim 40 \mathrm{~min}$. The effect of this is to cause the measured $x$ and $y$ centroids of an astronomical target to "wobble" by $\sim \pm 0.15$ " over the period of the heater cycle (one IRAC pixel spans an angle of $1.2^{\prime \prime}$ on the sky). In addition, spacecraft pointing exhibits a non-deterministic but secular drift of on average $0.35^{\prime \prime}$ per day. Thus measured fluxes can vary by $3-4 \%$ over the course of a $12 \mathrm{hr}$ staring observation. These intra-pixel gain variations are the largest source of signal-correlated noise in IRAC photometry.

Usually the variations are removed by fitting a surface to the science data themselves as a function of $x$ and $y$ centroid position ("self-calibration"), but this can result in the removal of astrophysical interesting signals. For example, an extrasolar planet ("exoplanet") light curve (plot of flux versus time as a planet orbits its parent star) may contain information on multiple timescales. Planetary phase, which varies as the side of the planet facing the observer changes, usually has a period equal to that of the orbit (for tidally locked systems); whereas primary or secondary eclipses are much shorter. Self-calibration works best when either (1) a part of the light curve is known to be free of planetary system variations or (2) a good model is available to fit both the planetary and instrument effects simultaneously. The first condition will not hold when measuring phase curves, since the full extent of the light curve will contain planetary variations (if detectable). As regards the second condition, a good model is usually only available when a target has been studied before, reducing the usefulness of Spitzer for characterizing new systems.

Spitzer Science Center, Infrared Processing and Analysis Center, California Institute of Technology, Mail Code 220-6, Pasadena, CA 91125. Send correspondence to JGI: Email: ingalls@ipac.caltech.edu; Phone: 16263958659

Space Telescopes and Instrumentation 2012: Optical, Infrared, and Millimeter Wave, edited by Mark C. Clampin, Giovanni G. Fazio, Howard A. MacEwen, Jacobus M. Oschmann, Jr., Proc. of SPIE Vol. 8442, 84421Y

(C) 2012 SPIE · CCC code: 0277-786/12/\$18 $\cdot$ doi: 10.1117/12.926947

Proc. of SPIE Vol. $844284421 Y-1$ 
It makes sense, then, to derive an independent calibration of the position-dependent effects. The simplest approach consists of sub-pixel mapping of (one or more) non-varying source(s) at very high spatial resolution. Until recently, it would have been extremely time-consuming to make the measurements necessary for such a calibration, because the area characterized would have to be about 1-3 pixels in diameter, to account for target acquisition pointing uncertainties (under blind pointing, Spitzer is incapable of positioning a target within a calibrated area to better than about 1 pixel) and drift. To cover even a few IRAC pixels at the desired resolution and to the precision required for correcting exoplanet measurements would require 1-3 months of observatory time.

In this paper, we describe a new technique for removing position-correlated noise, and greatly improving photometric precision, without using the data to be corrected. The method consists of (1) an adaptive positioning technique ("Peak-Up") that uses the Spitzer Pointing Calibration Reference Sensor (PCRS) to ensure that a target is placed within a small, well-calibrated "sweet spot" on a designated pixel; and (2) high spatial resolution low noise mapping of the photometric gain as a function of intra-pixel centroid position over the $0.5 \times 0.5$-pixel "sweet spot" region centered near the peak of response.

In the following section (Sec. 2), we describe our measurements to characterize the PCRS Peak-Up technique and to build the pixel gain maps. In Sec. 3 we examine the repeatability statistics after PCRS Peak-Up versus blind pointing, and describe the measured pixel gain maps. In Sec. 4 we quantitatively assess the benefit of applying the gain map to photometric measurement. In Sec. 5 we introduce an additional source of correlated noise, residual nonlinearity. In Sec. 6 we summarize our results and their implications.

\section{METHODOLOGY}

\subsection{Photometric Data}

All results described herein used images of point sources taken with the warm Spitzer IRAC arrays with bandpasses centered on $3.6 \mu \mathrm{m}$ ("Channel 1") and $4.5 \mu \mathrm{m}$ ("Channel 2"). Sources mainly consisted of standard flux calibration stars that are known not to vary in brightness; and known (variable) extrasolar planetary systems. The images have all been processed by the IRAC data reduction pipeline or a variant thereof. We processed further all basic calibrated data (BCD) images in the following ways.

1. We determined the centroids $\left(x_{\mathrm{cen}}, y_{\mathrm{cen}}\right)$ of the source images in pixel coordinates by measuring the intensityweighted mean positions:

$$
\begin{gathered}
x_{\mathrm{cen}}=\frac{\sum_{j, k}\left(I_{j k} j\right)}{\sum_{j, k} I_{j k}} \\
y_{\mathrm{cen}}=\frac{\sum_{j, k}\left(I_{j k} k\right)}{\sum_{j, k} I_{j k}} .
\end{gathered}
$$

Here $I_{j k}$ is the surface brightness in the pixel located at (column,row $)=(j, k)$, where the coordinates start at $(0,0)$ at the bottom left corner of the (full- or sub-) array. In these coordinates, a whole number value for $x_{\text {cen }}$ or $y_{\text {cen }}$ would mean that the target was at the center of a pixel. In practice the sums were truncated to a small region of the array around the expected target location.

2. We performed aperture photometry on the images, centering a 3-pixel radius circular aperture on the derived centroid locations $\left(x_{\mathrm{cen}}, y_{\mathrm{cen}}\right)_{i}$, integrating the flux inside the aperture, and subtracting a background flux measured in an annulus of inner radius 3 pixels and outer radius 7 pixels. We used the IDL astronomy library routine aper.pro*, which can measure flux in circular apertures on square pixels. The result was the flux of the source, $f_{i}$, which was usually in Jy.

\footnotetext{
*http://idlastro.gsfc.nasa.gov/ftp/pro/idlphot/aper.pro
} 
The resulting set of $\left\{\left(x_{\mathrm{cen}}, y_{\mathrm{cen}}, f\right)_{i}\right\}$ triplets formed the primary data used in our analysis. We also retained $t_{i}$, the time of measurement; (DN) ${ }_{i}$, the raw (uncalibrated) data number in the pixel of peak intensity (which is approximately proportional to the accumulated electron charge, or fluence, in a given measurement); and the uncertainties in the measured quantities $\left(\sigma_{x}, \sigma_{y}, \sigma_{f}\right)_{i}$.

\subsection{The PCRS Peak-Up Technique}

All observations (except the blind pointing comparison data discussed in Sec. 3.1) used the Spitzer PCRS PeakUp option to enhance the precision of positioning a source on a designated sub-pixel location. The Pointing Calibration and Reference Sensor operates in the visual part of the spectrum (505-595 nm) and its main function is to calibrate and remove the optical offset between the star trackers and the telescope. It can measure the centroids of stars in the $7.0 \mathrm{mag}<V<12.5 \mathrm{mag}$ range to within $0.14^{\prime \prime}$ ( $1 \sigma$ radial) and feed these data, in the form of a pointing correction, to the spacecraft pointing control system (PCS). The PCRS was used frequently in the Spitzer cryogenic mission with the Infrared Spectrograph $\left(\right.$ IRS $\left.^{6}\right)$. As we show below, PCRS Peak-Up allows us to repeatedly place a target within a preselected $0.5 \times 0.5$-pixel region in $98 \%$ of trials. This is the region over which we are building a high resolution map of the photometric gain.

\subsection{Deriving Photometric Gain Maps}

Early in the warm Spitzer mission, we built low resolution photometric gain maps of the nominal center pixels of the four available fields of view (FOVs): Channels 1 and 2, Full Array and Subarray. ${ }^{\dagger}$ These maps each covered an entire pixel and were used to correct stellar data as part of the overall flux calibration. Even though they were too coarsely sampled to use in $<1 \%$ precision staring photometry, these full pixel maps provided useful information on the structure of the intrapixel gain. In particular, we were able to derive a rough location of the peak of sensitivity, which is not the center of a pixel. For the 2 IRAC subarray FOVs, Channel 1 has its peak of sensitivity near $(x, y)=(15.20,15.02)$ and Channel 2 has its peak near $(x, y)=(15.12,15.09)$. Since the photometric gain over a pixel is roughly Gaussian in shape, putting a target near the peak of sensitivity serves two purposes: (1) it ensures that the $\mathrm{S} / \mathrm{N}$ is maximized; and (2) it minimizes the photometric noise due to response variations coupled with pointing jitter/wobble, since the response varies most slowly nearest the peak. Using PCRS Peak-Up combined with a commanded offset in array coordinates ${ }^{\ddagger}$, we are able to reliably place a target within a $0.5 \times 0.5$-pixel box centered on each of these locations, and so have begun mapping these $1 / 4$-pixel boxes at a high sampling rate $(>20$ samples contribute to each $0.002 \times 0.002$-pixel grid point in the "sweet spots" of the final maps).

For the high-precision gain maps, we have taken repeated photometric observations of the calibration stars KF09T1 (also known as TYC 4212-1074-1; 170 mJy at $3.6 \mu \mathrm{m}$ ) in Channel 1 and BD+67 1044 (also known as NPM1+67.0536; $480 \mathrm{mJy}$ at $4.5 \mu \mathrm{m}$ ) in Channel 2. We commanded 5 offset positions (in arcseconds) relative to the sensitivity peaks: $(-0.06,-0.06),(-0.06,+0.06),(0,0),(+0.06,-0.06)$, and $(+0.06,+0.06)$. For each offset position, we stared for about 40 minutes per Astronomical Observation Request (AOR; self-contained observation with PCRS Peak-Up at the beginning), accumulating subarray frames (exposures) of the targets at the rate of one per $0.4 \mathrm{~s}$ (Channel 1) and one per 0.1s (Channel 2). As we show below, commanded offsets are accurate to about 0.08 pixels $(1 \sigma$ radial), so we have let this intrinsic offsetting uncertainty, coupled with $\sim 0.1$-pixel pointing jitter and $\sim 0.3$-pixel pointing wobble (which has a period of $\sim 40$ minutes), convert the 5 discrete offsets into a stochastically determined set of $(x, y)$ pixel locations that uniformly cover the desired $0.5 \times 0.5$-pixel area.

To form the pixel photometric gain map (the "pmap"), we interpolated the random $\left\{\left(x_{\text {cen }}, y_{\text {cen }}, f\right)_{i}\right\}$ measurements onto a regular grid following Ballard et al, ${ }^{7}$ where the interpolation uses an error-weighted Gaussian kernel in $x$ and $y$ :

$$
\bar{F}(x, y)=\frac{\sum_{i<} f_{i} K_{i}}{\sum_{i<} K_{i}}
$$

where the kernel is $K_{i}(x, y)=O_{i}(x, y) / \sigma^{2}\left(f_{i}\right)$ [the kernels are weighted by the flux measurement errors, $\sigma\left(f_{i}\right)$ ], and we introduce the measurement occupation, the fractional contribution of the $i$ th data point to the map value

\footnotetext{
${ }^{\dagger}$ http://irsa.ipac.caltech.edu/data/SPITZER/docs/irac/calibrationfiles/pixelphase/

${ }^{\ddagger}$ http://irsa.ipac.caltech.edu/data/SPITZER/docs/irac/pcrs_obs.shtml
} 
at a given $(x, y)$ grid point:

$$
O_{i}(x, y) \equiv \exp \left\{-\left[\frac{\left(x-x_{i}\right)^{2}}{2 \delta_{x}^{2}}+\frac{\left(y-y_{i}\right)^{2}}{2 \delta_{y}^{2}}\right]\right\} .
$$

The " $i<$ " symbol in the sums indicates that the sums are truncated to include only that set of data points $\{i\}$ where $\left|x-x_{i}\right| \leq n \delta_{x}$ and $\left|y-y_{i}\right| \leq n \delta_{y}$. We usually take $n=10$. The width of the interpolating kernel is specified using $\delta_{x}$ and $\delta_{y}$, which we set equal to the finest scale resolved by Ballard et al, ${ }^{7} \delta_{x}=\delta_{y}=0.0043$ pixels. Note that, unlike Ballard et al, we are not building our map using the dataset to be calibrated, so measurements on or near the actual grid points themselves will contribute to the map.

One statistic that has proven useful to our analysis is the total occupation number, $\mathbf{O}$, which estimates the number of data points that influence the value of $\bar{F}(x, y)$ at a given grid point:

$$
\mathbf{O}(x, y)=\sum_{i<} O_{i}(x, y) \text {. }
$$

Finally, the uncertainty in a given mean $\bar{F}(x, y)$ is given by

$$
\begin{aligned}
\sigma_{\bar{F}}^{2} & =\sum_{i<}\left(\frac{\partial \bar{F}}{\partial f_{i}}\right)^{2} \sigma^{2}\left(f_{i}\right) \\
& =\frac{\sum_{i<} O_{i}(x, y) K_{i}(x, y)}{\left[\sum_{i<} K_{i}(x, y)\right]^{2}} .
\end{aligned}
$$

\subsection{Using the Pixel Gain Map to Calibrate Photometry}

To apply the gain map to photometry of general point sources, we normalized the map $\bar{F}(x, y)$ to a mean of unity by dividing by its average gridded value over the pixel:

$$
P(x, y)=\frac{\bar{F}(x, y)}{\sum_{1}^{N_{\text {grid }}} \bar{F}(x, y) / N_{\text {grid }}}
$$

In practice $\bar{F}(x, y)$ was computed on a grid dimensioned in 0.002 -pixel increments $\left(N_{\text {grid }}=500 \times 500\right.$ points $)$ covering the nominal subarray pixel for each array, i.e., pixel $(15,15)$. This allowed us two grid points per interpolating kernel half-width (0.0043 pixels). This normalized version, $P(x, y)$, referred to colloquially as "the p-map", can be interpolated to any location $\left(x_{j}, y_{j}\right)$ on the pixel, and photometric data $\left\{\left(f, x_{\text {cen }}, y_{\text {cen }}\right)_{j}\right\}$ can be corrected by dividing by $P\left(x_{j}, y_{j}\right)$. Since our Channel 1 and 2 p-maps are only well-sampled in the "sweet spot" regions, we use the occupation maps to ascertain whether a given point $(x, y)$ has sufficient sampling to make a high quality correction (see Sec. 3.2 below).

\section{RESULTS}

\subsection{PCRS Peakup Repeatability}

Figure 1 shows centroid measurements on the IRAC $4.5 \mu \mathrm{m}$ (Channel 2) subarray for the first 64 frames (averaged together) of 146 astronomical observation requests (AORs), taken for either calibration or scientific purposes. Dark circles show centroid positions on the IRAC $4.5 \mu \mathrm{m}$ array for the first exposures of 78 different exoplanet science AORs that used PCRS Peak-Up in the standard way, with the Spitzer gyroscopic Inertial Reference Unit (IRU) performing attitude determination during offset to IRAC ("incremental pointing" mode). Squares are the centroids for the first exposures of 20 AORs of calibration star BD+67 1044, taken with PCRS Peak-Up but using the Spitzer star tracker for attitude determination ("Observer" mode), which is accurate over long periods, but is extremely noisy for short offsets (i.e., offsets within the Spitzer focal plane). Triangles are the centroid positions for the first exposures of 48 AORs of calibration star HD 165459, taken directly after the initial slew to the star, without any Peak-Up refinement. Each plot symbol corresponds to different designated positions on the $4.5 \mu \mathrm{m}$ subarray. To assess the ability to return to the same intended spot after many trials, we subtracted 


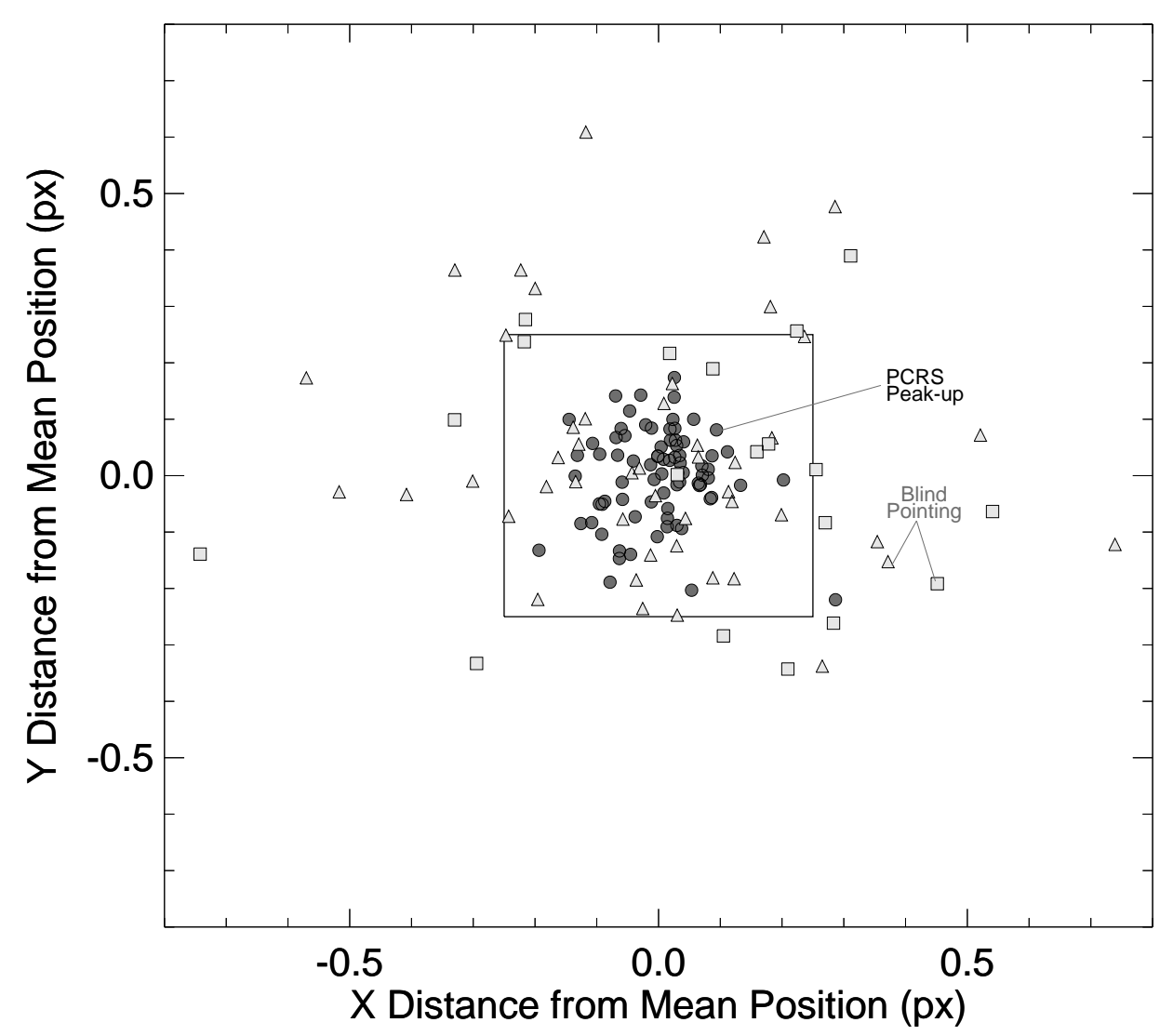

Figure 1. Demonstration of pointing repeatability using PCRS Peak-Up versus blind pointing. Each point represents the mean centroid of the first 64 frames of the following: 78 AORs (dark circles) beginning with PCRS Peak-Up followed by an offset to the $4.5 \mu \mathrm{m}$ subarray using gyros; 20 AORs (light squares) that also use PCRS Peak-Up, but using the star tracker for the subsequent offset to the $4.5 \mu \mathrm{m}$ subarray; 48 AORs (light triangles) taken without PCRS Peak-Up. Each set of symbols has had the mean position of the set subtracted. An $0.5 \times 0.5$-pixel square is overlaid on the plot, indicating the size of the predefined "sweet spot" region.

the mean value from each set of points. The two sets of light-colored points (squares and triangles), which we characterize as "blind pointing", have the same RMS, about 0.3 pixels. So evidently the noise in the star tracker masks any pointing improvement obtained from the Peak-Up. The dark-colored circles (PCRS Peak-Up followed by offset to IRAC using gyros) have an RMS of 0.08 pixels, showing a marked improvement in repeatability over blind pointing. PCRS Peak-Up mode placed a target within an $0.5 \times 0.5$-pixel "sweet spot" in 77 of 78 attempts, a $98 \%$ success rate. For blind pointing, only about $69 \%$ of attempts were successful; $10 \%$ of the trials were not even within the desired pixel!

\subsection{Pixel Photometric Gain Maps}

We display in Figure 2 maps of the Occupation number, $\mathbf{O}(x, y)$ (Eq. 5), and photometric gain, $P(x, y)$ (Eq. 7; the "p-map") as a function of centroid location for the nominal central pixels of the Channel 1 and 2 subarrays. The current maps are comprised of 453,800 individual photometric measurements at $3.6 \mu \mathrm{m}$ and 287,900 measurements at $4.5 \mu \mathrm{m}$. These maps are available online, together with an IDL software package to correct photometry. ${ }^{\S}$ As expected, the occupation maps inversely scale with the formal error in the p-map images (Eq. 6) (not shown),

\footnotetext{
${ }^{\S}$ http://irsa.ipac.caltech.edu/data/SPITZER/docs/dataanalysistools/tools/contributed/irac/iracpc_pmap_corr/
} 

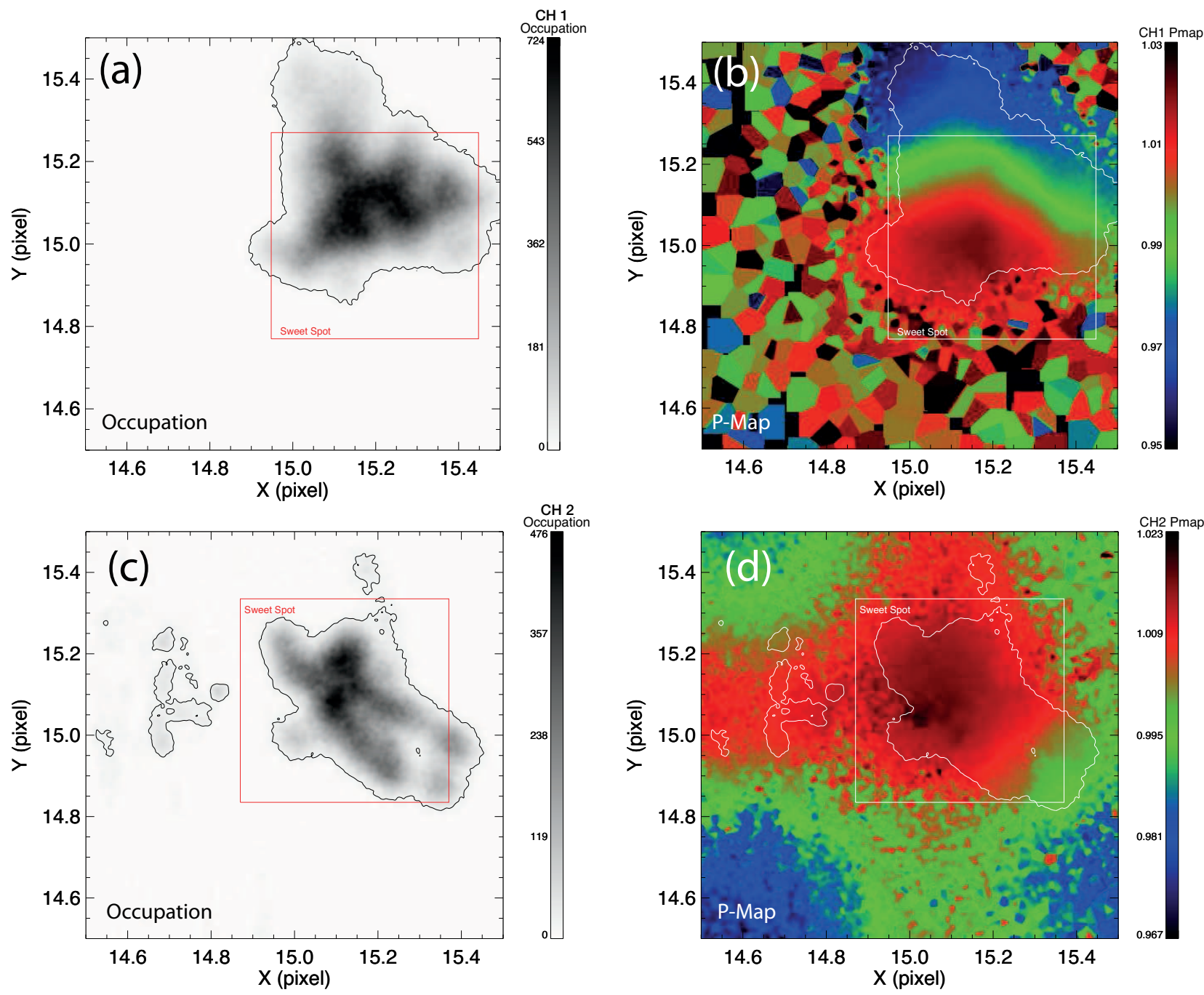

Figure 2. Maps of photometric response in the nominal center pixels of the IRAC 3.6 and $4.5 \mu$ m subarrays. Maps are gridded as a function of centroid location on 0.002 -pixel intervals $(500 \times 500$ sub-pixel locations). The plots show: (a) Channel 1 total occupation number, O (Eq. 5); (b) Channel 1 photometric gain ("p-pap"); (c) O for Channel 2; (d) p-map for Channel 2. Contours showing $\mathbf{O}=20$, and the nominal sweet spot $0.5 \times 0.5$-pixel box centered on the center of response are displayed on all frames.

so we adopt the occupation maps as a proxy for signal-to-noise in the p-map images. We find that, when $\mathbf{O}(x, y) \gtrsim 20$, the formal error in the (normalized) p-maps is $\sigma_{P}(x, y) \lesssim 10^{-3}$, a typical minimum requirement on exoplanet transit precision. So we define an occupation number of 20 as the threshold above which data are considered usable.

We overlay on the maps contours showing $\mathbf{O}(x, y)=20$. Inside of these contours the p-maps appear relatively smooth (but structured), whereas outside of them there is noticeable noise in the maps. Early in the Spitzer post-cryogenic mission Channel 2 pixel $(15,15)$ was mapped completely (but with very low sampling) using the same target as used in the current p-maps, and we include these data in Fig. 2 (d). This is why the Channel 2 map has detectable (albeit noisy outside $\mathbf{O}(x, y)=20$ ) gain variations across the entire pixel. Channel 1 was mapped fully as well, but with a different target than was used in the current maps (the target was changed to a fainter source for the current maps to minimize nonlinearity). Thus most of the $3.6 \mu \mathrm{m}$ data are of the sweet spot region only. Outside $\mathbf{O}(x, y)=20$, the Channel 1 occupation number becomes less than 1 for many grid 
points and the Channel 1 p-map obtains a very irregular appearance.

\section{USING THE TECHNIQUE TO REDUCE CORRELATED NOISE}

The PCRS Peak-Up plus p-map technique (referred to below simply as "the p-map technique") has been in operation for warm Spitzer staring mode observations since late 2011. We show here some results of applying the technique the sort of data on which they would be used in practice: light curves of extrasolar planetary systems.

\subsection{Cumulative distributions}

The goal of the p-map calibration is to eliminate, without using the data to be calibrated, noise that is correlated with the signal, and be left with the signal from the planetary system plus purely random noise that can be arbitrarily suppressed by averaging. In Fig. 3 we show cumulative distributions for the data of two systems, which measure the fraction of data that differs by a given percent from the mean. In addition to the original data, we display the Poisson noise limit, the data corrected using a polynomial surface in pixel $x$ and $y$ fit to the data themselves ("self-calibrated"), and data corrected using the independently-derived p-map. The noise limit was derived using a random number generator to produce a Poisson process with the same number of samples and the same mean value as the original dataset. The polynomial used for self calibration was 2 nd order in $x$ and $y$, but contained cross terms up to 4 th order in the combined coordinates (i.e., $x y^{2}, x^{2} y$, and $x^{2} y^{2}$ ). Panel (a) shows the cumulative distribution of 13,983 photometric data points taken for the HAT-P-17 exoplanetary system and panel (b) shows the data for 15,391 points taken for KOI 069, an as-yet unconfirmed candidate planetary system in the Kepler catalog. In both cases, the p-map technique brings $90 \%$ of the data to within $0.2 \%$ of the Poisson limit. In fact, the technique gives a reduction in the overall data scatter equal to (HAT-P-17) or better than (KOI 069) that obtained via self-calibration. Note that we have not removed any planetary system signal in either case, so if such a signal is present then even perfectly corrected data will not behave like Poisson noise.
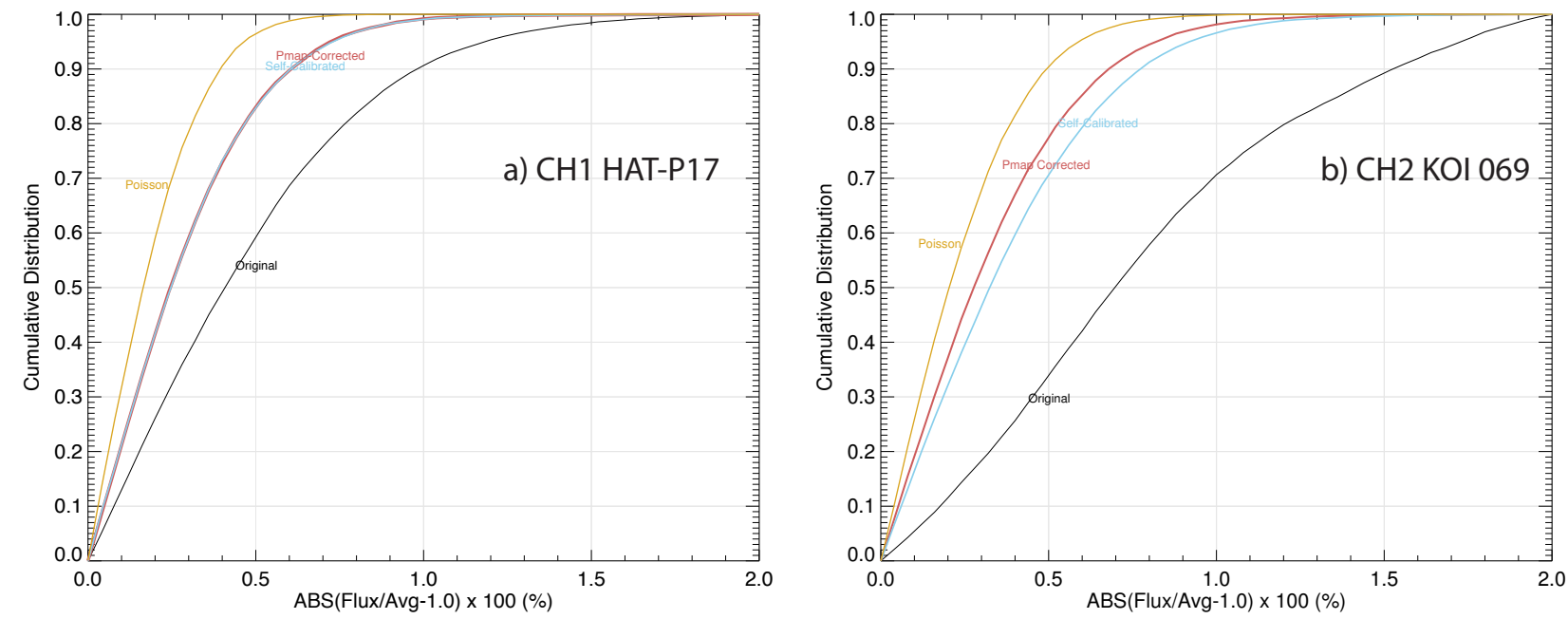

Figure 3. Cumulative distributions of data for two exoplanetary systems (candidate exoplanetary system, in the case of KOI 069), showing the fraction of data that differs by a given percent from the mean. The four curves show the original photometric measurements (black), those corrected by the p-map (red), those corrected by a 2nd order polynomial surface in pixel $x$ and $y$ ("self-calibrated") (blue), and the Poisson noise limit (yellow). (a) Channel 1 data for HAT-P-17; (b) Channel 2 data for KOI 069. 


\subsection{Light Curves}

Although cumulative distributions can assess the relative scatter in a dataset, they ignore temporal relationships. We use two additional statistics to demonstrate the reduction in signal-correlated noise in time series data: (1) signal averaging statistics and (2) the periodogram.

\subsubsection{Signal averaging statistics}

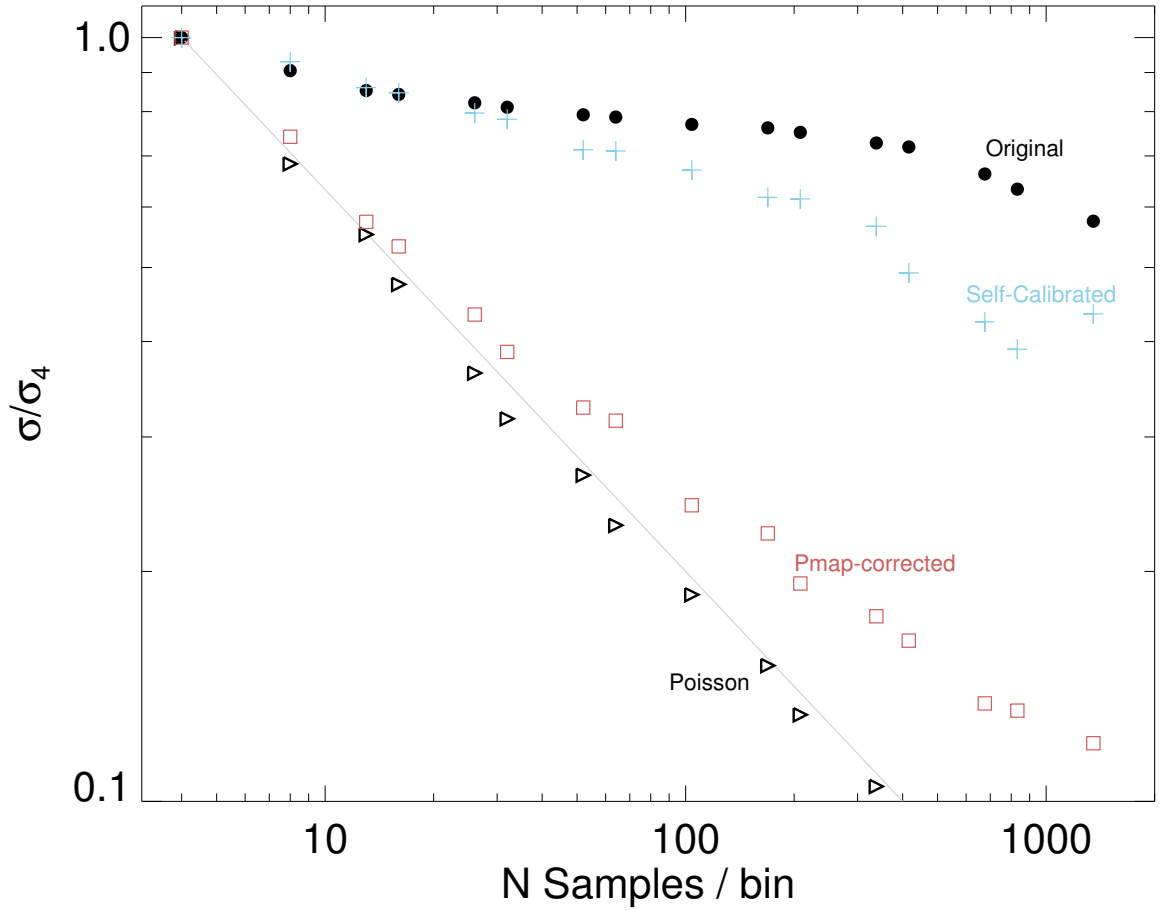

Figure 4. Signal averaging statistics for the KOI 069 light curves described in Fig. 3. Different symbols show the measured standard deviation, $\sigma$, in binned light curves, as a function of bin size, normalized by $\sigma_{4}$, the standard deviation for a 4-sample bin. Filled circles are the original data; "+" symbols are for self-calibrated data; squares are for p-map corrected data; and triangles are for a Poisson noise process. We show the theoretical Poisson noise expectation $\left(\propto N^{-1 / 2}\right)$ as a gray line.

To assess how well a technique succeeds at eliminating correlated noise, we can measure how well averaging, or binning, measurements together reduces the noise in the binned time series. Where the noise is purely random, its RMS should decrease as the square root of the number of samples in each bin. Figure 4 demonstrates noise reduction with binning as a function of bin size, using the KOI 069 Channel 2 light curve data. The triangular symbols show how the standard deviation in a purely random Poisson process decreases with bin size and the gray line plots the theoretical limit, $\propto 1 / N^{1 / 2}$. The original data (circles) show a very slow decrease in scatter with increasing number of samples per bin, indicating a high degree of correlated noise (or a very strong signal). The self-calibrated data show a somewhat improved falloff. The p-map corrected data behave extremely close to Poisson noise. Some non-random behavior remains; this could be additional noise, or an exoplanetary signal.

\subsubsection{The periodogram}

Since a major root cause of correlated noise in Spitzer light curves is periodic pointing wobble due to heater cycling, we can quantify the reduction in this type of noise using a periodogram.

Figure 5 shows the light curve of an exoplanetary system, with a parent star that is known to pulsate with a period of about 72 minutes. (The data are part of an unpublished Spitzer General Observer Program and 


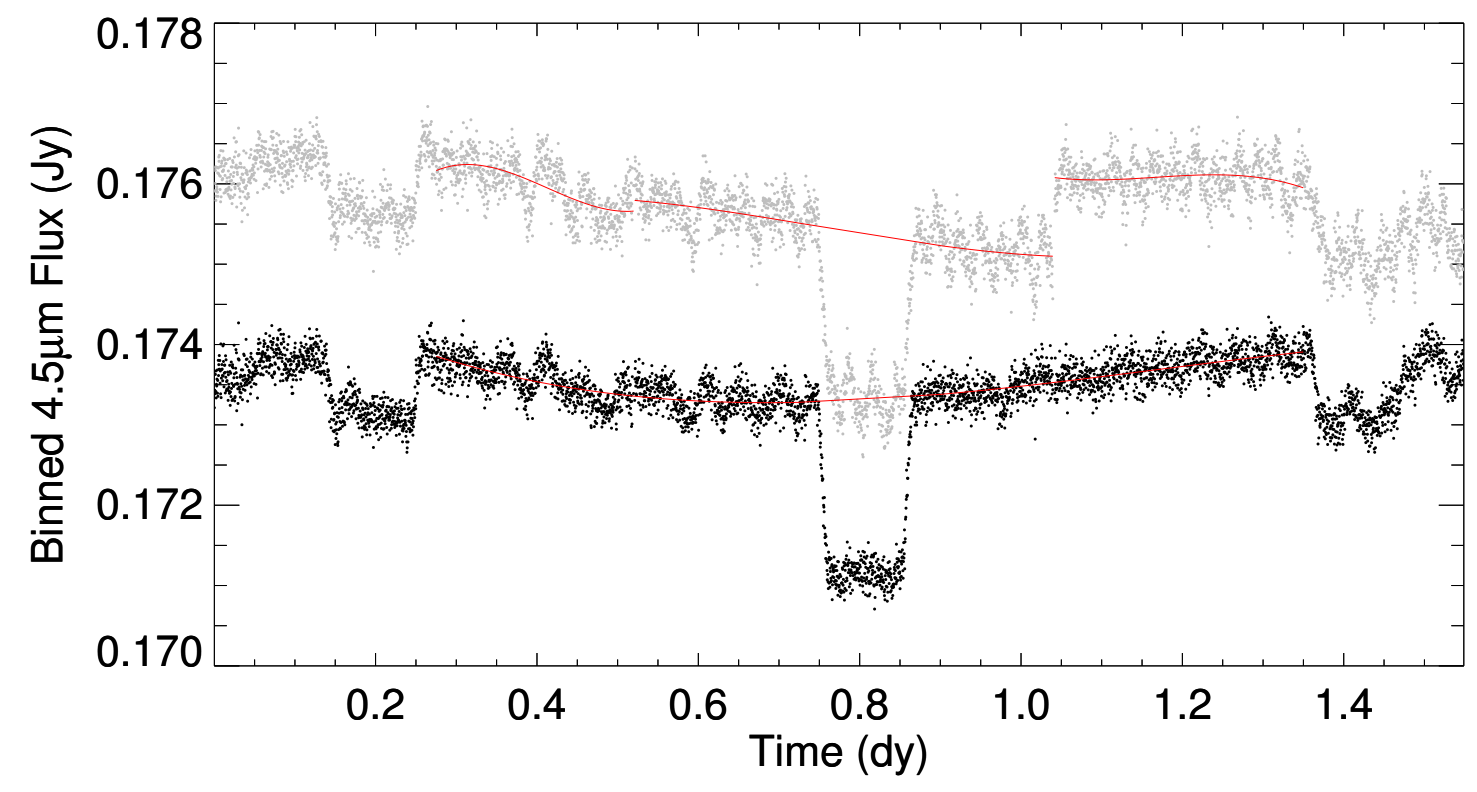

Figure 5. Light curve for a known extrasolar planetary system with a host star that pulsates. The two curves plot: Gray) the uncorrected photometric data; (Black) data corrected using the p-map. Red curves show 3rd order polynomial fits to the data outside of the transits. For the uncorrected data, we fit 3 curves, one per AOR. We fit only a single curve to the corrected data.

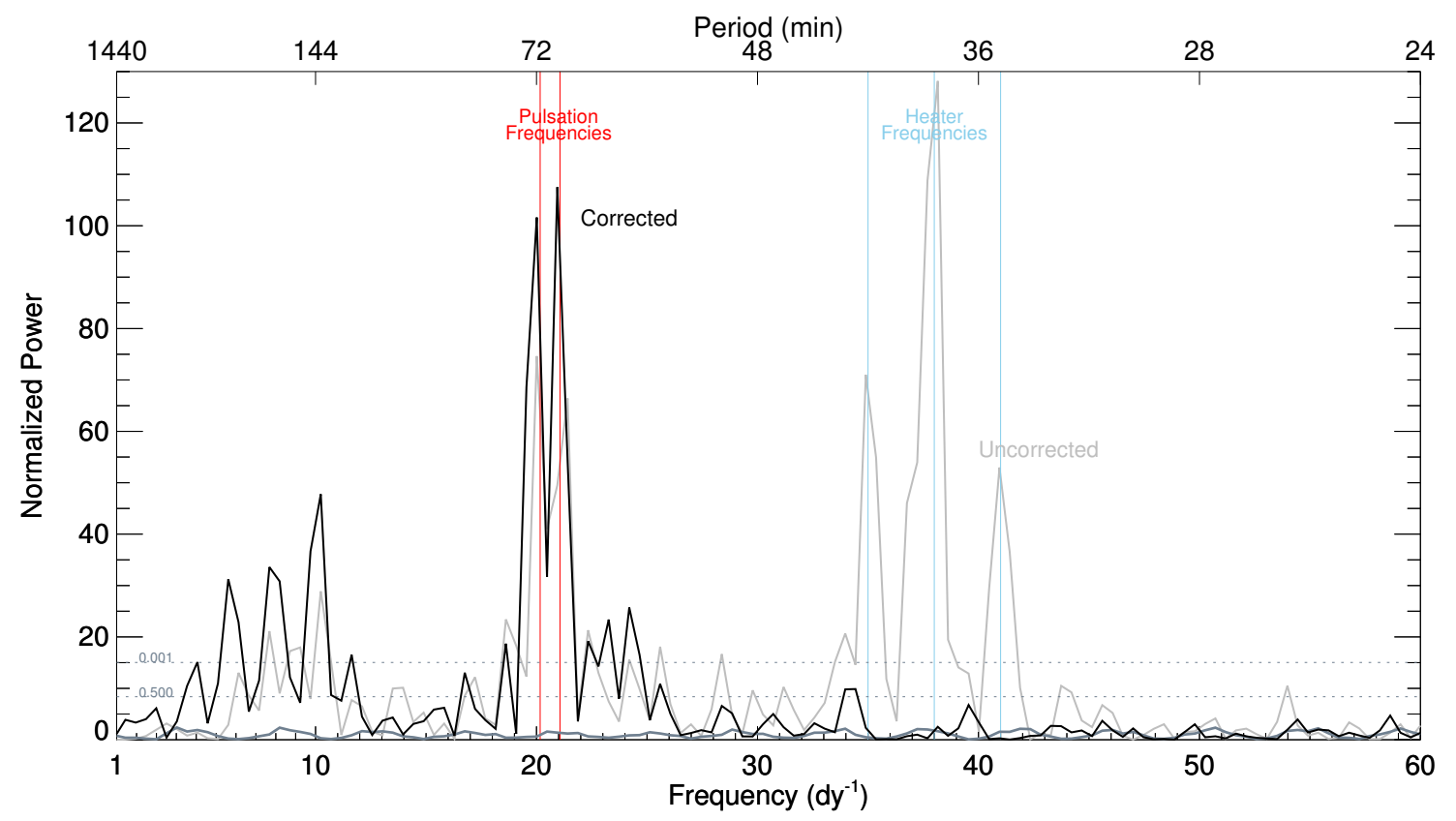

Figure 6. Lomb-Scargle normalized periodograms for uncorrected (gray) and p-map corrected (black) light curves shown in Fig. 5. Significance levels 0.5 and 0.001 are shown as horizontal dashed lines. We indicate the known stellar pulsation frequencies in red and the known heater cycle frequencies in blue. 
are displayed here for demonstration purposes only.) The gray points show the uncorrected photometry and the black points show the data after correction with the p-map. The measurements were comprised of 3 separate $\sim 12 \mathrm{hr}$ AORs executed back-to-back, with a PCRS Peak-Up at the beginning of each. Due to pointing drift during the second AOR, the third AOR shows a noticeably discontinuous jump in the flux after Peak-Up refreshes the position at the beginning. Correction by the p-map virtually eliminates this jump. The corrected light curve shows clearly the primary transit, two secondary transits, and a $\sim 0.5 \mathrm{mJy}$ amplitude planetary phase curve with a period equal to the entire orbit (the cool side of the tidally locked planet faces the observer during primary transit and the warm side of the planet faces the observer during secondary). Periodic signals of order 1 hour are visible in both light curves, but it is not easy to discern from the time series whether the signals are caused by the $\sim 40$ min pointing wobble due to heater cycling, or the $\sim 72$ min stellar pulsations.

To identify any periodic signals, we computed the Lomb-Scargle normalized periodogram for the aperture fluxes measured in the original and p-map corrected dataset. This kind of power spectrum is useful for data that are not regularly sampled, or contain gaps. To isolate only short ( $\sim 1$ hour $)$ timescales, we used only data outside of the transits and removed the phase variations by subtracting polynomial baselines fit to the data. To avoid the between-AOR jumps in the uncorrected data adding excess power to the periodogram, we fit three polynomials to the uncorrected data, one per AOR. Figure 6 shows the periodograms for the baseline-subtracted measurements. Significance levels 0.001 and 0.5 are shown as horizontal dashed lines. Peaks below the line labeled 0.5 are at least $50 \%$ likely to be noise, and peaks above the line labeled 0.001 are less than $99.9 \%$ likely to be noise. Three large peaks are seen in the uncorrected data, with frequencies known to correspond to the heater cycle. These peaks are almost completely removed after correction of the data by the p-map. Conversely, the amplitudes of the known stellar pulsation frequencies, which are significant in the uncorrected data, become

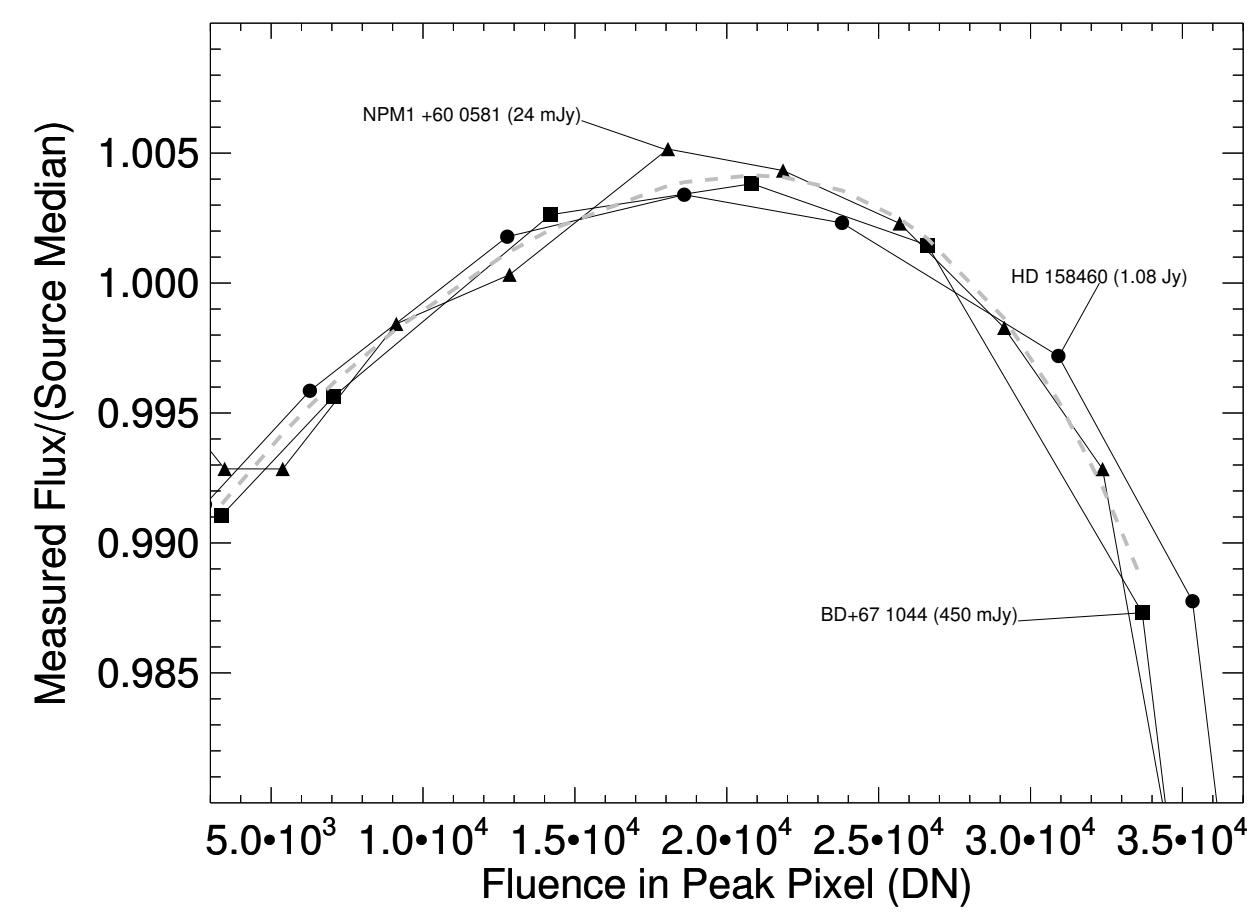

Figure 7. Demonstration of residual nonlinearity in IRAC $4.5 \mu \mathrm{m}$ photometry. Solid curves/filled symbols show the measured aperture flux, divided by the median over all observations, as a function of peak fluence (DN) for three calibration stars with different intrinsic fluxes. All data have been corrected for nonlinearity using the standard pipeline correction. Each symbol indicates the average (DN,flux) pair for a set of measurements taken with the same integration time. The dashed curve is a 4 th-order polynomial fit to the data. 
even more so after correction.

\section{CORRELATED NOISE FROM RESIDUAL NONLINEARITY}

The IRAC data reduction pipeline includes a correction for detector nonlinearity, which boosts the signal slightly at high electron fluence to ensure that count rate is proportional to flux. The correction was optimized for dithered observations, and has been averaged over multiple pixels across the 3.6 and $4.5 \mu$ m arrays. Furthermore, the linearization precision was never intended to be better than $\sim 1 \%$, consistent with quoted IRAC photometric precision. ${ }^{8}$ Thus it is reasonable to expect that staring mode data taken at the subarray sweet spots would have uncorrected residual nonlinear behavior manifesting as $\lesssim 1 \%$ variations in measured fluxes that correlate with fluence. Indeed, Fig. 7 shows how Channel 2 measured aperture flux varies as the source fluence increases, for three different targets with widely varying fluxes. When the measured flux is normalized by the source median over all fluence levels, the three sources show identical behavior: an inverted approximately parabolic curve (we have fit a 4th order polynomial to the data) with a total range of about $1.5 \%$. We refer to these variations as "residual nonlinearity".

Thus depending on where a measurement lies on the Fig. 7 curve, intra-pixel photometric gain variations for all data that pass through the IRAC pipeline may differ slightly from the p-map. Depending on the slope of the Fig. 7 relationship, the dynamic range of gain variations should increase and decrease with fluence. We do in fact see changes in the dynamic range of the intra-pixel response for observations with different mean DN levels, as shown in Fig. 8. The p-map data themselves are shown (open triangles). Comparison with the other targets shows as much as $1 \%$ variation in dynamic range from the low fluence p-maps $(\langle\mathrm{DN}\rangle \approx 2950)$ all the way to measurements with a nearly full well $(\langle\mathrm{DN}\rangle \approx 28000)$.

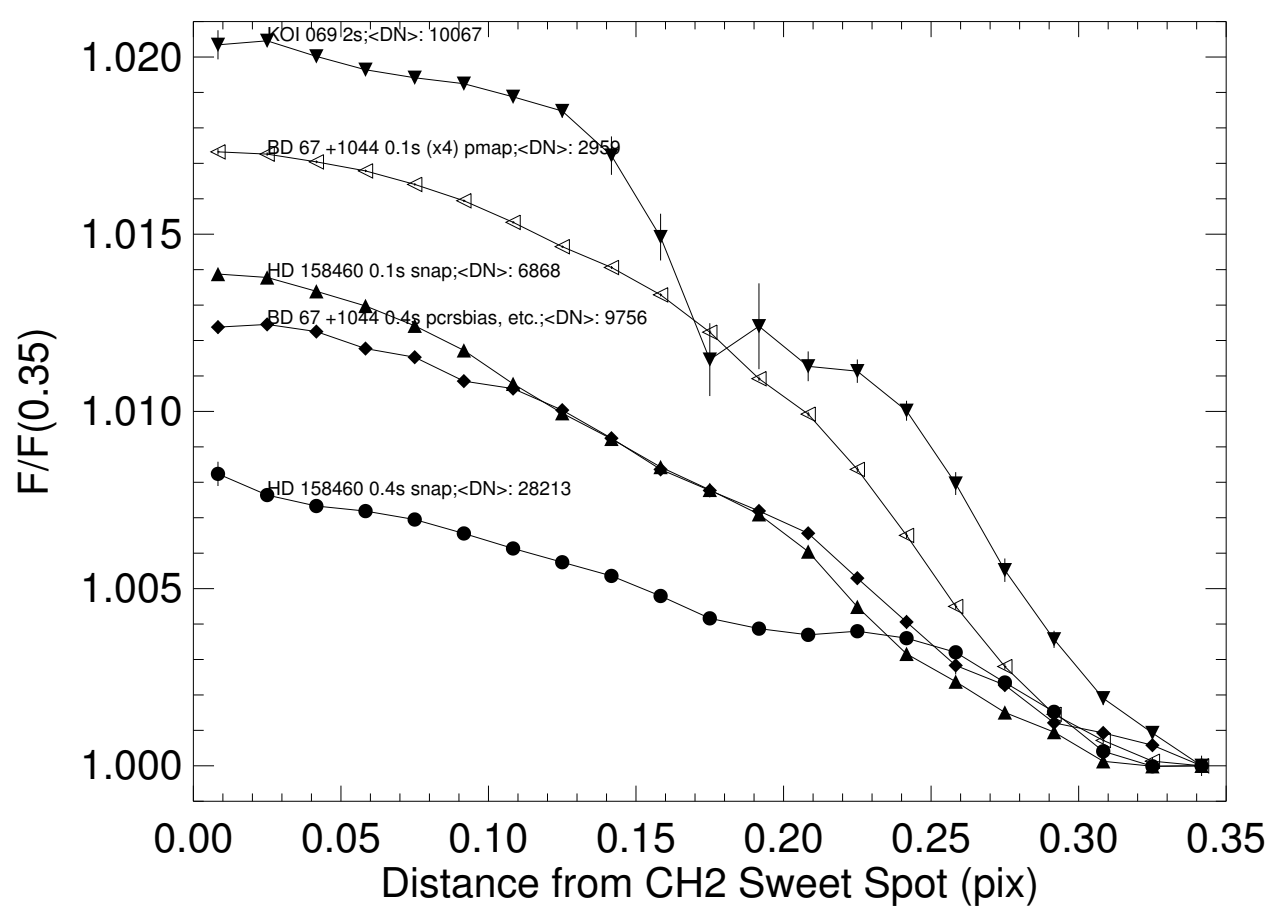

Figure 8. One-dimensional profiles of intra-pixel gain for experiments with different mean source fluence. Each symbol denotes a radial average of the measured aperture flux of a given target observed with a given exposure time, binned as a function of $r_{\text {sweet }}$, the distance from the nominal Channel 2 sweet spot center, normalized by the value at $r_{\text {sweet }}=0.35$. Error bars indicate the spread in the data in each bin. 
This additional source of correlated noise will prevent the existing p-map correction from fully calibrating all datasets (except for ones with similar well depth). We are currently still trying to understand the relationship between the residual nonlinearity and intrapixel gain (Figs. 7 and 8), but our goal is to eventually develop a gain correction that is a function of $x, y$, and $\mathrm{DN}$.

\section{SUMMARY}

We have described a technique for improving significantly photometric precision with warm Spitzer. The technique uses enhanced target acquisition via the PCRS Peak-Up and placement onto a pre-designated IRAC pixel "sweet spot", combined with a densely sampled high signal-to-noise map of the intrapixel gain across the sweet spot. This "p-map" technique has been in operation for staring mode observations since the end of 2011, and we have demonstrated the reduction in flux-correlated noise that results from its use. We are currently working on understanding an additional source of correlated noise, residual nonlinearity. These improvements will allow Spitzer to continue yielding unprecedented scientific discoveries in the realm of exoplanets and other fields of time domain astronomy, for as long as it remains operational.

\section{ACKNOWLEDGMENTS}

This work is based on observations made with the SpitzerSpace Telescope, which is operated by the Jet Propulsion Laboratory, California Institute of Technology under a contract with NASA.

\section{REFERENCES}

[1] Fazio, G. G., Hora, J. L., Allen, L. E., Ashby, M. L. N., Barmby, P., Deutsch, L. K., Huang, J.-S., Kleiner, S., Marengo, M., Megeath, S. T., Melnick, G. J., Pahre, M. A., Patten, B. M., Polizotti, J., Smith, H. A., Taylor, R. S., Wang, Z., Willner, S. P., Hoffmann, W. F., Pipher, J. L., Forrest, W. J., McMurty, C. W., McCreight, C. R., McKelvey, M. E., McMurray, R. E., Koch, D. G., Moseley, S. H., Arendt, R. G., Mentzell, J. E., Marx, C. T., Losch, P., Mayman, P., Eichhorn, W., Krebs, D., Jhabvala, M., Gezari, D. Y., Fixsen, D. J., Flores, J., Shakoorzadeh, K., Jungo, R., Hakun, C., Workman, L., Karpati, G., Kichak, R., Whitley, R., Mann, S., Tollestrup, E. V., Eisenhardt, P., Stern, D., Gorjian, V., Bhattacharya, B., Carey, S., Nelson, B. O., Glaccum, W. J., Lacy, M., Lowrance, P. J., Laine, S., Reach, W. T., Stauffer, J. A., Surace, J. A., Wilson, G., Wright, E. L., Hoffman, A., Domingo, G., and Cohen, M., "The Infrared Array Camera (IRAC) for the Spitzer Space Telescope," The Astrophysical Journal Supplement Series 154, 10-17 (Sept. 2004).

[2] Werner, M. W., Roellig, T. L., Low, F. J., Rieke, G. H., Rieke, M., Hoffmann, W. F., Young, E., Houck, J. R., Brandl, B., Fazio, G. G., Hora, J. L., Gehrz, R. D., Helou, G., Soifer, B. T., Stauffer, J., Keene, J., Eisenhardt, P., Gallagher, D., Gautier, T. N., Irace, W., Lawrence, C. R., Simmons, L., Van Cleve, J. E., Jura, M., Wright, E. L., and Cruikshank, D. P., "The Spitzer Space Telescope Mission," The Astrophysical Journal Supplement Series 154, 1-9 (Sept. 2004).

[3] Demory, B.-O., Gillon, M., Deming, D., Valencia, D., Seager, S., Benneke, B., Lovis, C., Cubillos, P., Harrington, J., Stevenson, K. B., Mayor, M., Pepe, F., Queloz, D., Segransan, D., and Udry, S., "Detection of a transit of the super-Earth 55 Cancri e with warm Spitzer," Astronomy and Astrophysics 533, 114 (Sept. 2011).

[4] Todorov, K. O., Deming, D., Knutson, H. A., Burrows, A., Sada, P. V., Cowan, N. B., Agol, E., Désert, J.-M., Fortney, J. J., Charbonneau, D., Laughlin, G., Langton, J., Showman, A. P., and Lewis, N. K., "Warm Spitzer Observations of Three Hot Exoplanets: XO-4b, HAT-P-6b, and HAT-P-8b," The Astrophysical Journal 746, 111 (Feb. 2012).

[5] Grillmair, C. J., Carey, S. J., Stauffer, J. R., Fisher, M. E., Olds, R., Ingalls, J. G., Krick, J. E., Glaccum, W. J., Laine, S., and Surace, J. A., "Pointing effects and their consequences for Spitzer IRAC exoplanet observations," in [Proc SPIE Vol 8448: Astronomical Telescopes and Instrumentation], 1-7 (July 2012).

[6] Houck, J. R., Roellig, T. L., Van Cleve, J., Forrest, W. J., Herter, T., Lawrence, C. R., Matthews, K., Reitsema, H. J., Soifer, B. T., Watson, D. M., Weedman, D., Huisjen, M., Troeltzsch, J., Barry, D. J., Bernard-Salas, J., Blacken, C. E., Brandl, B. R., Charmandaris, V., Devost, D., Gull, G. E., Hall, P., Henderson, C. P., Higdon, S. J. U., Pirger, B. E., Schoenwald, J., Sloan, G. C., Uchida, K. I., Appleton, 
P. N., Armus, L., Burgdorf, M. J., Fajardo-Acosta, S. B., Grillmair, C. J., Ingalls, J. G., Morris, P. W., and Teplitz, H. I., "The Infrared Spectrograph (IRS) on the Spitzer Space Telescope," The Astrophysical Journal Supplement Series 154, 18-24 (Sept. 2004).

[7] Ballard, S., Charbonneau, D., Deming, D., Knutson, H. A., Christiansen, J. L., Holman, M. J., Fabrycky, D., Seager, S., and A'Hearn, M. F., "A Search for a Sub-Earth-Sized Companion to GJ 436 and a Novel Method to Calibrate Warm Spitzer IRAC Observations," Publications of the Astronomical Society of the Pacific 122, 1341 (Oct. 2010).

[8] Hora, J. L., Carey, S., Surace, J., Marengo, M., Lowrance, P., Glaccum, W. J., Lacy, M., Reach, W. T., Hoffmann, W. F., Barmby, P., Willner, S. P., Fazio, G. G., Megeath, S. T., Allen, L. E., Bhattacharya, B., and Quijada, M., "Photometry using the Infrared Array Camera on the Spitzer Space Telescope," The Publications of the Astronomical Society of the Pacific 120, 1233 (Nov. 2008). 